\title{
Retrospective study of outcome in patients with scorpion sting using prazosin with/without dobutamine
}

\author{
L Venugopal', H Anilkumar², Dilip M Ram Pure ${ }^{3}$ L Suresh Kumar, Rajashekarappa G5 \\ ${ }^{1}$ Associate Professor, Dept of General Medicine, Mamata Medical College \& General Hospital, Khammam 507002 , Telangana, ${ }^{2}$ Associate \\ Professor, Dept of General Medicine, Raja Rajeshwari Medical College, Mysore Road, Bangalore 560074 , Karnataka, ${ }^{3}$ Professor and Head, \\ Dept of General Medicine, Mamata Medical College \& General Hospital, Khammam 507002, Telangana, ${ }^{4}$ Post Graduate Trainee, Dept of \\ General Medicine, Mamata Medical College \& General Hospital, Khammam 507002, Telangana, ${ }^{5}$ Professor, Dept of General Medicine, \\ Mamata Medical College \& General Hospital, Khammam 507002, Telangana
}

A B S TR A C T

Introduction: Scorpion sting is a major public health problem in India with severe life threatening complications. Toxin can cause autonomic overstimulation leading to hypertension, arrhythmias and pulmonary edema with left ventricular dysfunction. Prazosin has been the ideal antidote for patients with mild to moderate envenomation. In patients with severe envenomation, dobutamine is found to be very useful to prevent morbidity and mortality. Materials and Methods: Retrospective study was done on patients admitted from March 2011 to November 2013 with a history of scorpion bite and adults aged between 20-60 yrs were included. All the patients were subjected to grading of envenomation as mild, moderate and severe degree. Prazosin was given to all 80 patients and dobutamine was given to those who did not show response to prazosin. Results: Majority of patients were in the age group of 31-40 years followed by 21-30 years. Mean age was $31.23 \pm 7.23$ years. Fifty four patients $(67.5 \%)$ were male and twenty six $(32.5 \%)$ were female. 28 patients had mild envenomation, 36 had moderate envenomation and 16 had severe envenomation. 83.33\% of patients responded to dobutamine infusion in prazosin resistant cases in our study. Conclusion: Early use of dobutamine should be considered in patients with severe envenomation with left ventricular dysfunction to prevent morbidity and mortality. 2D echo has to be considered to assess left ventricular dysfunction and need for early use of dobutamine.

Access this article online

Website:

http://nepjol.info/index.php/AJMS DOI: 10.3126/ajms.v7i2.13051 E-ISSN: 2091-0576 P-ISSN: 2467-9100

Key words: Scorpion sting, Prazosin, Dobutamine, Outcome, 2DECHO

\section{INTRODUCTION}

Scorpion sting is a life threatening medical emergency in rural areas of India. The toxin causes release of neurotransmitters such as acetylcholine \& catecholamines, leading to manifestations of sympathetic and parasympathetic overactivity. Severe life threatening cardio respiratory effects including severe hypertension, shock and bradyarrhythmias, and pulmonary edema with or without myocardial dysfunction can occur.

There are no standard guidelines or protocols for the management of scorpion envenomation. The present study was undertaken to know the outcome of the use of prazosin and dobutamine (in prazosin resistant cases).

\section{METHODS AND MATERIALS}

A retrospective study was done on patients admitted from March 2011 to November 2013 with a history of scorpion bite. Adults aged between 20-60 yrs were included in the study. Ethical committee approval was taken.

According to the initial assessment, all the patients were subjected to grading of envenomation.

\section{Grading of envenomation}

Mild envenomation: Local pain, tingling + sweating and vomiting. No cardiovascular symptoms. 
Moderate envenomation: Profuse sweating, sinus tachycardia or bradycardia, tall ' $T$ ' waves on ECG, reduced LV function on 2D Echo, hypertension, hypotension, LVF (Killips class I \& II) + Local signs.

Severe envenomation: Pulmonary edema + signs of moderate envenomation.

Total of 86 patients were taken of which 6 patients were excluded. Prazosin was given to all 80 patients and observed for response. 18 patients did not show response after 24 hours and were given dobutamine.

\section{Treatment protocol followed}

Tetanus Toxoid

Local treatment with 2\% Xylocaine

Prazosin

In patients with Left ventricular dysfunction

Diuretics and Dopamine

Patients who did not respond to above Treatment

Dobutamine Intra venous (IV)

\section{RESULTS}

Out of the 86 patients 80 patients fulfilled the inclusion criteria. They were divided into two groups, those given prazosin alone and those given both prazosin and dobutamine.

Table 1 explains the Age and gender distribution of both groups. Majority of patients were in the age group of 31-40 years followed by 21-30 years. Mean age was $31.23 \pm 7.23$ years. Fifty four patients $(67.5 \%)$ were male and twenty six $(32.5 \%)$ were female.

Table 2 explains the background characteristics of the two groups. Forty six patients were hospitalized during day time (6am to $6 \mathrm{pm}$ ) whereas 34 patients were admitted during evening \& night time (6pm to $6 \mathrm{am})$. Majority of patients were affected during summer season.

All the patients presented with stings on the exposed areas of the body upper or lower extremities. 48 patients presented with scorpion sting on the upper extremities whereas, 32 in lower extremities.

Twenty patients presented within 2 hours of bite, 53 patients came between 2-12 hours, whereas 7 patients presented after 12 hours of bite. Most common presenting features are sweating and pain.

Patients with bite during the night time, those who presented more than 12 hours after the bite and those with severe degree of envenomation were resistant to treatment with prazosin and received dobutamine IV.

Table 3 explains the relation between times elapsed before admission, severity and the outcome among both the groups. Out of 7 patients who presented more than 12 hours, 2 patients died.

In the present study, $83.33 \%$ of patients responded to dobutamine infusion in prazosin resistant cases.

\begin{tabular}{|c|c|c|c|c|c|}
\hline \multirow[t]{2}{*}{$\begin{array}{l}\text { Age } \\
\text { (years) }\end{array}$} & \multicolumn{2}{|c|}{$\begin{array}{c}\text { Prazosin } \\
(n=62)\end{array}$} & \multicolumn{2}{|c|}{$\begin{array}{l}\text { Prazosin+Dobutamine } \\
(n=18)\end{array}$} & \multirow{2}{*}{$\begin{array}{c}\text { Total } \\
\mathbf{n}\end{array}$} \\
\hline & Males & Females & Males & Females & \\
\hline $21-30$ & 15 & 6 & 4 & 1 & 26 \\
\hline $31-40$ & 18 & 8 & 5 & 3 & 34 \\
\hline $41-50$ & 6 & 5 & 2 & 1 & 14 \\
\hline $51-60$ & 2 & 2 & 2 & 0 & 6 \\
\hline Total & 41 & 21 & 13 & 5 & 80 \\
\hline
\end{tabular}

\begin{tabular}{|c|c|c|}
\hline Characteristics & $\begin{array}{l}\text { Prazosin } \\
(n=62)\end{array}$ & $\begin{array}{c}\text { Prazosin+Dobutamine } \\
(n=18)\end{array}$ \\
\hline \multicolumn{3}{|l|}{ Gender } \\
\hline Males (\%) & 76.9 & 23.1 \\
\hline \multicolumn{3}{|c|}{ Presenting features } \\
\hline Pain & 56 & 12 \\
\hline Sweating & 52 & 12 \\
\hline \multicolumn{3}{|l|}{ Season } \\
\hline May-June & 15 & 5 \\
\hline Sep-Oct & 26 & 7 \\
\hline \multicolumn{3}{|l|}{ Time of bite } \\
\hline 6 am-6 pm & 40 & 6 \\
\hline 6 pm-6 am & 22 & 12 \\
\hline \multicolumn{3}{|l|}{ Site of bite } \\
\hline Upper limbs & 40 & 8 \\
\hline Lower limbs & 22 & 10 \\
\hline \multicolumn{3}{|l|}{ Grading } \\
\hline Mild & 28 & 0 \\
\hline Moderate & 34 & 2 \\
\hline Severe & 0 & 16 \\
\hline \multicolumn{3}{|c|}{ Time before admission } \\
\hline$<2 \mathrm{hrs}$ & 17 & 3 \\
\hline $2-12 \mathrm{hrs}$ & 43 & 10 \\
\hline$>12 \mathrm{hrs}$ & 2 & 5 \\
\hline
\end{tabular}

\begin{tabular}{lcccc}
\multicolumn{3}{l}{ Table 3: Severity and outcome of envenomation } \\
\hline Grade & $\begin{array}{c}\text { Time lapse } \\
\text { before } \\
\text { admission (hrs) }\end{array}$ & $\begin{array}{c}\text { Treatment given } \\
\text { Prazosin }\end{array}$ & $\begin{array}{c}\text { Dobutamine } \\
\text { with prazosin }\end{array}$ \\
\hline Mild & $<12$ & 26 & 0 & 0 \\
Moderate & $>12$ & 2 & 0 & 0 \\
Severe & $<12$ & 34 & 0 & 0 \\
& $>12$ & 0 & 2 & 0 \\
& $<12$ & 0 & 13 & 1 \\
\hline
\end{tabular}




\section{DISCUSSION}

The mechanism of cardio-toxicity in scorpion sting is multi factorial. Scorpion sting releases a neurotoxin which alters voltage gated sodium and potassium channels leading to enhanced autonomic stimulation. This leads to excess release of catecholamines causing hypertension and a transient phase of increased contractility due to excess alpha and beta receptor stimulation. There is diminished systolic performance in addition to the catecholamine effect. The combination of myocardial ischemia, excessive catecholamine effect, cardiac arrhythmia, and increased oxygen demand may result in acute myocardial ischemia and infarction. ${ }^{1-4}$

Prazosin, an adrenergic alpha -1 blocker is most suitable in antagonizing the toxic effect of Scorpion venom. It reduces the pre-load and left ventricular impedance without rise in heart rate and rennin secretion. Prazosin acts as a physiological antidote to scorpion venom and reverses blood pressure and pulmonary edema.,

Dobutamine infusion also improves cardiac output, blood pressure, tissue oxygenation, and clinical outcome significantly. It acts mainly by blocking beta mediated. It is useful in acute pulmonary edema in patients with severe hypotension. ${ }^{7,8}$

Our study shows that all the patients with severe envenomation were refractory to treatment with prazosin and required dobutamine, whereas only 2 out of 36 with moderate envenomation required dobutamine and none with mild envenomation. Hence, patients presenting with severe envenomation are more likely to be refractory to prazosin and may require dobutamine very early.

Five $(71.4 \%)$ patients out of 7 who presented after 12 hours of bite were refractory to prazosin and needed dobutamine, $10(18.8 \%)$ out of 53 patients presenting between $2-12$ hours needed dobutamine and only 3 (15\%) out of 20 presenting within 2 hours needed dobutamine. Also 2 out of 3 patients with severe envenomation who presented after 12 hours expired in spite of receiving dobutamine, while only one patient out of 13 with severe envenomation who presented between 2-12 hours expired. So, patients presenting very late after 12 hours are more likely to require dobutamine early.

Six $(13 \%)$ patients out of 46 who had scorpion bite during the daytime were refractory to prazosin, whereas $12(35.3 \%)$ out of 34 patients who had bite during night needed dobutamine. Thus, patients who were bitten during the night are more likely to present late and are more likely refractory to prazosin. Dobutamine may be needed early in these patients.
Eight (16.6\%) out of 48 patients who were bitten in the upper limbs required dobutamine while 10 (31.25\%) out of 32 patients who had bite on lower limbs required dobutamine. Patients with bites on lower limbs are more likely to be unaware of the bite and thus may present lately with features of severe envenomation and thus need dobutamine very early.

In the present study, 20\% patients were prazosin resistant which was correlating with Suvarna Patil et $\mathrm{al}^{9}$ which. $83.33 \%$ of patients responded to dobutamine infusion in prazosin resistant cases in our study and Suvarna Patil et al ${ }^{9}$ reported $92.5 \%$ response with dobutamine infusion in prazosin.

\section{CONCLUSION}

Prazosin is an ideal antidote in cases of mild to moderate scorpion envenomation. However, in patients with severe envenomation with left ventricular dysfunction early use of dobutamine should be considered to prevent morbidity and mortality. In addition to patients with severe envenomation, in patients with moderate envenomation presenting late, 2D echo has to be considered to assess left ventricular dysfunction and need for early use of dobutamine.

\section{REFERENCES}

1. Bawaskar HS and Bawaskar PH. Management of cardiovascular manifestations of poisoning by the Indian red scorpion (Mesobuthus Tamulus). British Heart J.1992; 68: 478-480.

2. Gueron M and Weizman S. Catecholamines and myocardial damage in scorpion sting. Amer. heart J. 1968; 25: 716-717.

3. Gueron M, stern J and Cohen W. Severe myocardial damage and heart failure in scorpion sting. Am J Cardiol 1967; 19: 719-726.

4. Gwee MCE, Nirtthanan S, Khoo H, Gopalkrishnakone P, Kini MR and Cheah LS. Autonomic effects of some scorpion venoms and toxins. Clinical experimental Pharmacology and Physiology 2002; 29: 795-801.

5. Bawaskar HS and Bawaskar PH. Prazosin therapy and scorpion envenomation. J Assoc Physicians India 2001; 49:121-123.

6. Bawaskar HS and Bawaskar PH. Prazosin in management of cardiovascular manifestations of scorpion sting. Lancet 1986; II: 510-511.

7. Elatrous S, Nouira S, Besbes-Ouanes L, Boussarsar M, Boukef R, Marghli and Abroug F. Dobutamine in severe scorpion envenomation. Chest 1999; 116: 748-752.

8. Hakim A, Sahnoun Z, Kassis M, Bouaziz M, Zeghal KM, and Kallel $H$. Beneficial effects of high dobutamine doses on hemodynamic perturbations induced by Buthus occitanus tunetanus venom in rats. Med Sci Monit 2009; 15(3): 71-74.

9. Patil SN. A Retrospective Analysis of a Rural Set up Experience with Special Reference to Dobutamine in Prazosin-Resistant Scorpion Sting Cases. JAPI 2009; 57: 301-304. 